\title{
Orléans - 20-22 rue Porte Madeleine/3 rue de la Grille
}

$n^{\circ} 064484$

Philippe Salé et Dominique Canny

\section{(2) OpenEdition \\ Journals}

Édition électronique

URL : http://journals.openedition.org/adlfi/14330

ISSN : 2114-0502

Éditeur

Ministère de la culture

Référence électronique

Philippe Salé et Dominique Canny, «Orléans - 20-22 rue Porte Madeleine/3 rue de la Grille », ADLFI.

Archéologie de la France - Informations [En ligne], Centre, mis en ligne le 20 mars 2015, consulté le 20 avril 2019. URL : http://journals.openedition.org/adlfi/14330

Ce document a été généré automatiquement le 20 avril 2019

(c) Ministère de la Culture et de la Communication, CNRS 


\title{
Orléans - 20-22 rue Porte Madeleine/3 rue de la Grille
}

$n^{\circ} 064484$

\author{
Philippe Salé et Dominique Canny
}

Lien Atlas (MCC) :

http://atlas.patrimoines.culture.fr/atlas/trunk/index.php?

ap_theme=DOM_2.01.02\&ap_bbox=1.874;47.813;1.949;47.933

1 La réalisation d'un projet immobilier sur sous-sol est à l'origine de l'intervention archéologique menée au 20/22 rue Porte Madeleine appelé aussi le 3 rue de la Grille. L'opération fait suite à une évaluation positive réalisée en 1998. L'opération de fouille a été effectuée aux mois de juillet et de septembre 1999 sur une superficie d'environ $2000 \mathrm{~m}$ .

2 Le site, localisé à l'ouest du centre historique de la ville, à environ $600 \mathrm{~m}$ au nord du cours actuel de la Loire, se place sur le rebord du plateau calcaire, en dehors du castrum à environ $300 \mathrm{~m}$ à l'ouest de celui-ci.

3 À environ $100 \mathrm{~m}$ à l'est du site, se trouve la place de la Croix-Morin, où la rue des Carmes arrivant de l'est se scinde en deux axes de direction très proches : la rue Porte Saint-Jean, en direction de Châteaudun/Le Mans au nord et la rue Porte Madeleine, au sud, en direction de Tours. Cette dernière est réputée être un axe antique.

L'opération au 20/22 rue Porte Madeleine est la quatrième intervention archéologique réalisée récemment dans le secteur ouest de la ville.

5 L'occupation la plus précoce du site est matérialisée par un four domestique attribuable à la fin de la période gauloise ( $\mathrm{e}^{\mathrm{er}} \mathrm{s}$. av. J.-C.). Cette découverte, qui peut paraître isolée et anecdotique, s'ajoute aux structures gauloises fouillées au n ${ }^{\circ} 18$ de la rue Porte Saint-Jean en 1998). Aussi, leur présence dans le quartier permet de reconsidérer les hypothèses formulées sur la nature, l'étendue et l'importance de l'occupation gauloise à Orléans. 
S'agit-il d'une extension urbaine ou d'une occupation isolée à proximité de l'agglomération (habitat, artisanat, exploitation agricole)?

6 Entre la fin du $\mathrm{I}^{\mathrm{er}} \mathrm{s}$. av. J.-C. et le début du $\mathrm{I}^{\mathrm{er}}$ s. apr. J.-C, les informations sur l'occupation sont plus rares. Deux structures se rapportent à l'époque augustéenne et un espace de circulation, orienté nord-sud, est installé aux environs des années 50 apr. J.-C. Il reprend peut-être les limites d'un tracé plus ancien (de la fin de l'époque gauloise ?) qui est resté difficilement perceptible à la fouille. La voie, qui est encadrée de palissades, apparaît comme un élément ordonnateur du paysage. Des regroupements de fosses sont installés le long du passage et leur organisation s'adapte au cours du $\mathrm{I}^{\mathrm{er}} \mathrm{s}$. à un changement d'orientation de la voie. Elle est désormais orientée sud-ouest/nord-est et les palissades qui lui sont associées sont abandonnées. Cette réorientation a lieu dans la seconde moitié $\mathrm{du} \mathrm{I}^{\mathrm{er}} \mathrm{s}$. apr.J.-C. L'absence presque totale de stratification ne permet pas d'affiner la datation du changement d'orientation de la voie.

L'occupation du site est continue entre le milieu du $\mathrm{I}^{\mathrm{er}} \mathrm{s}$. et le début du $\mathrm{II}^{\mathrm{e}} \mathrm{s}$. apr. J.-C. Le lieu est investi de nombreuses fosses quadrangulaires aménagées. Elles sont groupées et forment des unités réparties le long d'une voie.

8 Les groupes sont séparés les uns des autres par des espaces vides. L'interruption des palissades semble correspondre à des passages ou à des limites. L'endroit semble divisé en trois parties distinctes : un groupe de fosses au sud, un autre au centre et le troisième au nord. Peut-être faut-il y voir l'expression d'une division en parcelles, en ateliers ou en unités de production?

9 Les vestiges issus de la fouille permettent d'envisager les activités artisanales pratiquées sur le gisement au $\mathrm{I}^{\mathrm{er}}$ s. apr. J.-C.

10 La première activité est liée au textile. Elle est essentiellement représentée par des poids de métiers à tisser découverts dans des fosses aménagées.

Le mobilier osseux a livré des vestiges, peu nombreux, mais révélateurs d'une pratique de la taille de l'os (déchets de taille, produits finis et semi-finis). Il est tentant d'évoquer l'existence d'une activité de tabletterie sans être affirmatif. Le site s'inscrit peut être à proximité immédiate de ce type d'artisanat.

Ces deux activités renforcent l'idée d'un secteur artisanal qui est peut-être spécialisé, et situé dans une frange urbaine, le long d'un axe de circulation.

À partir du II $\mathrm{e}$ s. apr. J.-C., la densité d'occupation apparaît plus faible. Il ne reste que deux ateliers de travail sur le site. Les autres sont abandonnés et ne restent plus que quelques fosses et puits. L'occupation dans le quartier apparaît plus lâche et les groupements de fosses disparaissent. Les voies semblent toujours utilisées car aucune structure ne vient les recouper. Ces transformations correspondent à une disparition progressive jusqu'au III ${ }^{\mathrm{e}} \mathrm{s}$. apr. J.-C. des activités artisanales dans ce secteur de la ville. Ce phénomène traduit vraisemblablement un déplacement des activités artisanales vers un autre espace de la ville. Il peut cependant également s'agir d'une transformation de la forme des structures. L'enfouissement des cellules de travail, constaté aux $\mathrm{I}^{\mathrm{er}}$ et $\mathrm{II}^{\mathrm{e}} \mathrm{s}$. n'est peut-être plus d'actualité.

14 À compter du II $\mathrm{S}$ apr. J.-C., plus aucune trace d'activité n'est décelable, en dehors de la présence de terres végétales. Il semble qu'il faut y voir la transformation de ce secteur en une zone à vocation agricole ou pastorale. 
15 Il faut attendre l'extrême fin du Moyen Âge pour que le quartier connaisse un mouvement durable d'organisation. C'est à ce moment que naît la trame urbaine encore visible actuellement dans ce secteur de la ville. Une réoccupation du site a lieu à la période moderne. Elle est représentée par un lot de fosses et de puits. Un four de potier a été repéré et les archives confirment sa présence. Ce four utilisé de 1807 à 1827 par D. Vernois contribue à compléter les connaissances sur une des activités pratiquées dans le quartier à partir du XIX ${ }^{\mathrm{e}} \mathrm{s}$.

\section{INDEX}

Index géographique : Centre, Loiret (45), Orléans

Mots-clés : four, fosse, occupation gauloise, artisanat, voirie operation Fouille d'évaluation (EV) 